\title{
QUALIDADE DE VIDA DE MÉDICOS INTENSIVISTAS: UM ESTUDO TRANSVERSAL
}

\author{
QUALITY OF LIFE OF INTENSIVE DOCTORS: A TRANSVERSAL STUDY
}

\section{Kelly Jéssica Trindade Costa ${ }^{a^{*}}$, Denison Santos Silva ${ }^{b^{*}}$, Alline Oliveira da Silva Porto ${ }^{c^{*}}$, Fernanda Gomes de Magalhães Soares Pinheiro ${ }^{\mathrm{d}^{* *}}$, Gustavo Melo Rios Souza ${ }^{\mathrm{e}^{* * *}}$, Natália Sampaio Carvalho Bonfim ${ }^{\mathrm{f} * * *}$, Eduardo Enrico Ferrari Nogueira ${ }^{\mathrm{g}^{* * * * *}}$, Damião da Conceição Araújo ${ }^{\text {h** }}$}

\author{
akelly.tc@hotmail.com, bdenisansil@hotmail.com, callineoliveiramed@gmail.com, dfernandagmsoares@gmail.com, \\ egustavomelorios@yahoo.com.br, fnatysampaio_@hotmail.com, geduardodete@terra.com.br, hdamiao.araujo92@gmail.com \\ *Universidade Tiradentes - Aracaju (SE), Brasil \\ ** Universidade Federal de Sergipe - São Cristóvão (SE), Brasil \\ *** Hospital São José - Aracaju (SE), Brasil \\ **** Faculdade de Ciências Médicas de Campina Grande - Campina Grande (PB), Brasil \\ ***** Hospital Primavera - Aracaju (SE), Brasil
}

Data de recebimento do artigo: 29/06/2017

Data de aceite do artigo: 03/08/2017

\section{RESUMO}

Introdução: $\mathrm{O}$ ambiente da unidade de terapia intensiva desencadeia estresse nos profissionais médicos, afetando a qualidade de vida desses profissionais. Objetivo: Verificar a qualidade de vida dos médicos que trabalham em unidades de terapia intensiva no município de Aracaju, Sergipe. Métodos: Estudo observacional do tipo transversal, realizado em doze unidades de terapia intensiva do município de Aracaju, Sergipe, com uma amostra de 131 médicos. Foi aplicado um questionário para caracterização do perfil sociodemográfico e o World Health Organization's quality of life-bref (WHOQOL-bref). Os dados foram armazenados e analisados com o auxílio do programa Statistical Package for Social Science versão 21.0. Resultados: A qualidade de vida (QV) dos médicos foi considerada ruim (68,9\%). As alteraçóes nos domínios físicos, psicológicos, das relações sociais e do meio ambiente do WHOQOL-bref demonstraram influência distinta na QV dos médicos. Outras variáveis como tempo de formação, carga horária de trabalho e faixa etária também se associaram com o desfecho. Conclusão: Os médicos que trabalham nas unidades de terapia intensiva do munícipio de Aracaju possuem qualidade de vida ruim. Este estudo suscita a necessidade de que mais pesquisas sejam realizadas com essa temática.

Palavras-chave: Qualidade de vida; unidades de terapia intensiva; profissionais médicos; saúde do trabalhador.

\section{ABSTRACT}

Introduction: The environment of the intensive care unit triggers stress in medical professionals, affecting the quality of life of these professionals. Objective: To verify the quality of life of doctors working in intensive care units in the city of Aracaju, Sergipe. Materials and Methods: A cross-sectional observational study was performed at twelve intensive care units in the city of Aracaju, Sergipe, Brazil, with a sample of 131 doctors. A questionnaire was applied to characterize the sociodemographic profile and the World Health Organization's Quality of Life-Bref (WHOQOL-bref). The data were stored and analyzed with the help of the Statistical Package for Social Science program version 21.0. Results: Doctors quality of life was considered poor (68.9\%). Changes in the physical, psychological, social, and environmental domains of the WHOQOL-bref demonstrated a distinct influence on the QoL of physicians. Other variables such as training time, workload and age were also associated with the outcome. Conclusion: The doctors who work in the intensive care units of the municipality of Aracaju, Sergipe, have poor quality of life. This study raises the need for more research to be done on the subject.

Keywords: Quality of life; intensive care units; medical professionals; worker's health. 


\section{Introdução}

A unidade de terapia intensiva (UTI) desenvolveu-se para oferecer assistência aos pacientes em estado grave de saúde, proporcionando a redução dos índices de morbidade e mortalidade para diversas doenças ${ }^{1}$.

A atuação do médico é primordial na UTI. Entretanto, a rotina laboral, o estresse, a carga horária de trabalho, o acúmulo de vínculos empregatícios, o convívio constante com o sofrimento, a dor, o risco de morte dos pacientes, a superlotação de leitos, a falta de recursos humanos e de materiais são fatores desencadeadores para o desgaste físico, mental e psicológico que interferem, sobretudo, na qualidade de vida $(\mathrm{QV})$ desses profissionais ${ }^{2-4}$.

Nesse contexto e de acordo com a definição de QV pela Organização Mundial de Saúde (OMS) como "a percepção da pessoa sobre sua posição na vida dentro do contexto dos sistemas culturais e de valores nos quais ela vive e em relação a suas metas, expectativas pessoais e preocupaçóes" ", a avaliação da QV dos profissionais médicos é tema relevante, não apenas no que diz respeito à promoção da saúde, mas por influenciar na relação médico-paciente e no convívio com os familiares e colegas de trabalho ${ }^{3}$.

Destarte, objetivou-se com este estudo verificar a qualidade de vida dos médicos que trabalham em unidades de terapia intensiva no município de Aracaju, Sergipe, Brasil.

\section{Metodologia}

\section{Desenho e contexto do estudo}

Trata-se de um estudo observacional do tipo tranversal, aprovado pelo Comitê de Ética em Pesquisa em Seres Humanos da Universidade Tiradentes/UNIT sob protocolo CAAE no 47394015.5.0000.5371. Do total de quinze UTI, distribuidas por onze estabelecimentos de prestaçóes de serviços de saúde no estado de Sergipe ${ }^{6}$, a pesquisa contemplou como universo doze, que estáo localizadas em hospitais públicos e privados do município de Aracaju.

\section{Participantes}

A amostra foi não probabilística por conveniência, constituída de 131 médicos. Os critérios utilizados para o recrutamento e a inclusáo dos sujeitos na pesquisa foram: 1) ser médico que atua em unidades de terapia intensiva; 2) trabalhar em regime de plantão ou diarista; e 3) possuir ou náo especialidade na área de UTI. Como critério de exclusão utilizou-se os questionários que não obtivessem preenchimento adequado e/ou ausência da assinatura do Termo de Consentimento Livre e Esclarecido.

\section{Variáveis e instrumentos}

As variáveis investigadas de interesse para o estudo foram: 1) dependente: QV dos médicos; 2) independentes: idade, gênero, estado civil, anos completos de término da graduação, especialidade, local de trabalho, carga horária laboral, área de atuação e domínios físico, psicológico, relaçôes sociais e meio ambiente do WHOQOL-bref.

Os instrumentos utilizados foram um questionário para caracterização dos aspectos sociodemográficos elaborado pelos autores e o World Health Organization's Quality of Life-Bref(WHOQOL-bref). O WHOQOLbref é um instrumento recomendado pela OMS, adaptado, aplicado e validado para o Brasil e inclui quatro domínios relacionados à $\mathrm{QV}$ : físico, psicológico, relações sociais e meio ambiente ${ }^{7}$.

\section{Sistemática de coleta de dados}

A coleta de dados ocorreu durante o período de fevereiro a abril de 2016. Os pesquisadores compareceram nas UTI para identificar os profissionais médicos por intermédio da gerência hospitalar. Após a aplicação dos critérios de elegibilidade, os profissionais foram visitados no seu dia de trabalho para que fossem realizadas as orientaçóes e os esclarecimentos sobre o objetivo da pesquisa e fosse solicitada a participação por anuência verbal e assinatura do Termo de Consentimento Livre e Esclarecido. Posteriormente, foi entregue um envelope com os instrumentos de coleta de dados.

\section{Análise estatística}

Os dados foram armazenados e analisados pelo programa Statistical Package for Social Science (SPSS), versão 21.0 para Windows. Inicialmente, realizou-se a estatística descritiva dos dados. As variáveis numéricas foram calculadas por meio das medidas de tendência central e de dispersão. As variáveis categóricas foram apresentadas em frequência absoluta e relativa. $\mathrm{O}$ escore do WHOQOL-bref foi calculado usando a sintaxe recomendada pela $\mathrm{OMS}^{7,8}$.

Utilizou-se o teste do Qui-quadrado de Pearson para a associação entre as variáveis categóricas. Os domínios 
do WHOQOL-bref foram categorizados em valores baixos e valores altos em relação ao valor da média da amostra geral, para o uso na análise de regressão logística múltipla. Dessa forma, foi calculado o Odds Ratio (OR) e os respectivos intervalos de confiança de $95 \%$. Para todo o estudo, foi considerado o valor de $\mathrm{p}<0,05$ estatisticamente significante.

\section{Resultados}

A análise descritiva dos dados revelou idade média de 41 anos $( \pm 8,9)$. Quanto às características sociodemográficas, o total de $52,7 \%$ era do sexo feminino, $68,7 \%$ eram casadas, $67,9 \%$ atuantes na UTI adulto, $74,0 \%$ como plantonistas, $61,8 \%$ com o tempo de formação de 15 anos e 49,0\% com carga horária maior que 90 horas (Tabela 1).

Tabela 1: Características do perfil sociodemográfico dos médicos intensivistas, Aracaju, Sergipe, 2017.

\begin{tabular}{|c|c|c|c|}
\hline Varáveis & Categorias & $\mathbf{n}$ & $\%$ \\
\hline \multirow{2}{*}{ Gênero } & Masculino & 62 & 47,3 \\
\hline & Feminino & 69 & 52,7 \\
\hline \multirow{4}{*}{ Estado civil } & Solteiro & 26 & 19,8 \\
\hline & Casado & 90 & 68,7 \\
\hline & Divorciado & 13 & 9,9 \\
\hline & Viúvo & 2 & 1,5 \\
\hline \multirow{6}{*}{ Especialidade } & Neonatologia & 17 & 13,0 \\
\hline & Terapia Intensiva & 16 & 12,2 \\
\hline & Pediatria & 16 & 12,2 \\
\hline & Cardiologia & 15 & 11,5 \\
\hline & Clínica Geral & 14 & 10,7 \\
\hline & Outros & 53 & 40,4 \\
\hline \multirow{2}{*}{ Local de trabalho } & UTI & 89 & 67,9 \\
\hline & UTIN* & 42 & 32,1 \\
\hline \multirow{2}{*}{ Área de trabalho } & Plantonista & 97 & 74,0 \\
\hline & Diarista & 34 & 26,0 \\
\hline \multirow{3}{*}{ Tempo de formaçáo } & 3 anos & 30 & 23,0 \\
\hline & 5 anos & 20 & 15,2 \\
\hline & 15 anos & 81 & 61,8 \\
\hline \multirow{4}{*}{ Carga horária } & $12-24$ & 12 & 9,1 \\
\hline & $30-36$ & 19 & 14,5 \\
\hline & $40-60$ & 36 & 27,4 \\
\hline & $>90$ & 64 & 49,0 \\
\hline Total & & 131 & 100 \\
\hline
\end{tabular}

* UTIN: Unidade de Terapia Intensiva Neonatal.

Fonte: Elaborada com dados da pesquisa dos autores, 2016.

O cálculo do WHOQOL-bref revelou os escores descritos na Tabela 2. Considerou-se a escala de 0 a 100 para determinar os valores. Contudo, a média geral da amostra para cada domínio foi utilizada como parâmetro para identificar a QV ruim (escore menor que a média) e QV boa (escore maior que a média). O total de 68,9\% dos médicos possui QV ruim, com parâmetros iguais para todos os domínios.

Tabela 2: Escores do WHOQOL-bref conforme QV global e domínios, Aracaju, Sergipe, 2017.

\begin{tabular}{lcc} 
Variável/Categoria & Média/dp & p ${ }^{\dagger}$ \\
QV global & $69,9 \pm 10,9$ & $<0,01$ \\
Ruim (n/\%) & $90(68,7)$ & \\
Boa (n/\%) & $41(31,3)$ & \\
Domínio físico & $73,4 \pm 13,2$ & 0,00 \\
Ruim (n/\%) & $72(54,9)$ & \\
Boa (n/\%) & $59(45,1)$ & \\
Domínio psicológico & $69,2 \pm 12,9$ & $<0,01$ \\
Ruim (n/\%) & $76(58,1)$ & \\
Boa (n/\%) & $55(41,1)$ & \\
Domínio das relaçôes sociais & $71,0 \pm 17,8$ & $<0,01$ \\
Ruim (n/\%) & $76(58,1)$ & \\
Boa (n/\%) & $55(41,1)$ & \\
Domínio do meio ambiente & $66,0 \pm 11,1$ & 0,03 \\
Ruim (n/\%) & $66(50,3)$ & \\
Boa (n/\%) & $65(49,7)$ & \\
\hline
\end{tabular}

$\dagger$ Teste de Qui-quadrado de Pearson ( $\mathrm{p}<0,05$ ).

Fonte: Elaborada com dados da pesquisa dos autores, 2016.

A análise dos dados da Tabela 3 descreve a influência da alteração dos domínios do WHOQOL-bref em relação ao desfecho de QV ruim dos intensivistas. O domínio das relações sociais (OR: 2,4) e o psicológico (OR: 12,0) se destacam quanto aos valores associados.

Tabela 3: Análise de regressão logística múltipla dos domínios do WHOQOL-bref em relação ao desfecho de QV ruim dos médicos.

\begin{tabular}{lccc} 
Variável/categoria & OR* $^{*}$ & IC 95\%* & p \\
\hline QV ruim & & & \\
Domínio físico & 11,3 & $2,87-44,98$ & 0,00 \\
Domínio psicológico & 12,0 & $3,42-42,79$ & $<0,01$ \\
Domínio das relaçóes sociais & 21,4 & $5,05-91,22$ & $<0,01$ \\
Domínio do meio ambiente & 6,5 & $1,87-22,66$ & 0,03 \\
\hline
\end{tabular}

*Teste de regressão logística múltipla $(\mathrm{p}<0,05)$, OR: Odds ratio (razão de chances), IC 95\%: intervalo de confiança; †Teste de Qui-quadrado de Pearson $(\mathrm{p}<0,05)$.

Fonte: Elaborada com dados da pesquisa dos autores, 2016.

Os fatores associados com o desfecho da QV ruim apresentaram valores distintos conforme as categorias das variáveis analisadas (Tabela 4). Destaca-se, entretanto, que a carga horária maior que 90 horas (OR: 17,27), 
o tempo de formação maior que 15 anos (OR: 13,23) e a faixa etária de 48 a 69 anos (OR: 2,9) foram os que mais influenciaram o desfecho.

Tabela 4: Fatores associados com a QV ruim dos médicos intensivistas, Aracaju, Sergipe, 2017.

\begin{tabular}{lccc} 
Variável/categoria & QV ruim & p ${ }^{\dagger}$ \\
Carga horária & & & \\
IC $(\mathbf{9 5 \%})^{*}$ & & $<0,01$ \\
$30-24$ & 1,68 & $0,25-8,11$ & \\
$40-36$ & 2,88 & $1,17-11,27$ & \\
$>90$ & 8,25 & $3,19-26,59$ & \\
Tempo de formaçáo & 17,27 & $3,68-81,14$ & \\
3 anos & & & $<0,01$ \\
5 anos & 4,12 & $1,96-28,64$ & \\
15 anos & 8,40 & $1,96-28,64$ & \\
Gênero & 13,23 & $2,36-74,10$ & \\
Masculino & & & 0,03 \\
Feminino & 0,6 & $0,27-1,38$ & \\
Idade & 1,0 & $0,42-2,41$ & \\
$26-47$ & & & 0,00 \\
$48-69$ & 1,2 & $0,57-2,86$ & \\
\hline
\end{tabular}

*Teste de Regressão logística múltipla $(\mathrm{p}<0,05)$, OR: Odds ratio (razão de chances), IC: intervalo de confiança de 95\%; †Teste de Qui-quadrado de Pearson $(\mathrm{p}<0,05)$.

Fonte: Elaborada com dados da pesquisa dos autores, 2016.

\section{Discussão}

Neste estudo, o perfil sociodemográfico revelado foi o de adultos jovens, do gênero feminino, casados, especialistas em outras áreas da medicina, que atuam na UTI em regime de plantonista. O tempo de formação dos profissionais foi em média de 15 anos, e a carga horária de trabalho com média de 36,6 horas semanais. Os achados corroboram com outras pesquisas ${ }^{9-11}$.

A QV envolve questóes que podem alterar a percepção do sujeito, os sentimentos e os comportamentos, bem como a condição de saúde. $\mathrm{O}$ instrumento mais utilizado para mensurar a QV dos indivíduos, World Health Organization Questionnaire for Quality of Life Brief Form (WHOQOL-bref), que foi elaborado pela OMS, avalia a QV conforme os seguintes domínios: físico, psicológico, relações sociais e meio ambiente. Entretanto, a OMS não definiu o valor mínimo e máximo de QV aceitável. As análises devem ser realizadas com base na amostra para comparar os valores da média de cada domínio e determinar os fatores associados ${ }^{5,7}$.

A QV dos médicos de Aracaju, neste estudo, foi considerada ruim $(68,7 \%)$, uma vez que a maior parte obteve escore abaixo da média amostral. Para os domínios da $\mathrm{QV}$, ambos foram considerados com alteração: físico $(54,9 \%)$, psicológico $(58,1 \%)$, relaçóes sociais $(58,1 \%)$ e meio ambiente $(50,3 \%)$. Além disso, a análise de regressão logística revelou a razão de chances $(\mathrm{OR})$ de cada um dos domínios como fatores de risco, quando alterados, para a QV: físico (OR: 11,3), psicológico (OR: 12,0), relaçóes sociais (OR: 21,4) e meio ambiente (OR: 6,5).

O domínio físico tem relação com necessidades humanas básicas por estar relacionado com dor física, energia para as atividades, sono e desempenho de atividades de vida diárias e instrumentais, evidenciando que os agravos físicos interferem na $\mathrm{QV}^{12,13}$.

No que concerne à alteração no domínio psicológico, das relaçóes sociais e do meio ambiente, estes resultados estão relacionados ao aspecto de que o exercício da medicina provoca desgaste psicológico e modifica o convívio social e interpessoal, em razão do estresse ocupacional, da necessidade de maior tempo para o exercício da função nas instituiçóes de saúde, da responsabilidade social, do contato com o sofrimento de pacientes e familiares, bem como das condiçóes insa $\neg$ tisfatórias de trabalho ${ }^{14}$.

Outros fatores que se associaram ao desfecho da QV ruim foram a carga horária maior que 90 horas (OR:17,27), o tempo de formação maior que 15 anos (OR: 13,23) e a faixa etária de 48 a 69 anos (OR: 2,9). Estudos demonstram evidências de que o tempo de formação e a carga horária de trabalho está associada com a predisposiçấo à exaustão emocional, desencadeando, sobretudo, o esgotamento físico e psicológico, uma vez que pode ocorrer redução dos recursos biopsicossociais para enfrentar as situaçóes e os conflitos vivenciados no trabalho, bem como a dificuldade em encontrar energia suficiente para desempenhar as atividades diárias no âmbito da UTI ${ }^{10,15,16}$. A faixa etária como fator associado pode ser explicada devido ao aparecimento de questóes sociais, culturais e de saúde ${ }^{17}$.

O estudo da QV vem sendo aplicado nos ambientes e nas organizações de prestação de assistência à saúde, e sua finalidade é verificar indicadores no contexto social, no processo saúde-doença e no ambiente de trabalho, a fim de nortear a construção de políticas públicas e tornar os ambientes de trabalho mais saudáveis. Entretanto, a maior parte das pesquisas avalia a QV relacionando-a com pacientes portadores de doenças ${ }^{18,19}$ e profissionais da enfermagem ${ }^{20,21}$, uma vez que doenças relacionadas ao estresse, como a síndrome de burnout, afetam significativamente os profissionais de enfermagem. A população de profissionais médicos que trabalham em UTI ou em ambientes críticos de cuidado tem sido pouco explorada no que concerne à dimensão da QV, com limitaçôes para a discussão dos achados na população deste estudo. 


\section{Conclusão}

A QV dos médicos de Aracaju nas doze UTI estudadas foi considerada ruim. Os domínios físico, psicológico, das relaçóes sociais e do meio ambiente, bem como o tempo de formação, a carga horária de trabalho e a faixa etária se associaram com o desfecho.

Os pesquisadores deste estudo recomendam que mais pesquisas sejam realizadas para abordar a QV dos médicos no ambiente da UTI, uma vez que estas encontram-se escassas na base dados. Sergipe é o menor estado brasileiro e dispóe de cerca de $0,9 \%$ de cobertura de leito de UTI por população ${ }^{6}$. Portanto, conhecer os cenários das diferentes regiôes do Brasil e os perfis profissionais é necessário para melhorar os processos de trabalhos, a assistência à saúde, a satisfaçáo pessoal e profissional e, consequentemente, propor melhoria da QV.

\section{Referências}

1. Lacerda JC, Barbosa AP, Cunha AJLA. Perfil profissional do intensivista pediátrico no estado do Rio de Janeiro, sudeste do Brasil. Rev bras ter intensiva. 2011;23(4):462-9.

2. Kirchhof ALC, Magnago TSBS, Camponogara S, Griep RH, Tavares JP, Prestes FC, et al. Condiçóes de trabalho e características sócio-demográficas relacionadas à presença de distúrbios psíquicos menores em trabalhadores de enfermagem. Texto \& contexto enferm. 2009;18(2):215-23.

3. Acioli Neto ACF, Araújo RC, Pitangui ACR, Menezes LC, França EET, Costa EC, et al. Qualidade de vida e nível de atividade física de profissionais de saúde de unidades de terapia intensiva. Rev Bras Ativ Fís Saúde. 2013;18(6):711-9.

4. Baasch D, Laner AS. Os significados do trabalho em unidades de terapia intensiva de dois hospitais brasileiros. Ciênc saúde coletiva. 2011;16(Supl 1):1097-1105.

5. World Health Organization. Global status report on road safety 2013: supporting a decade of action. Luxembourg: WHO; 2013.

6. Associaçáo de Medicina Intensiva Brasileira. Medicina intensiva: contribuiçóes formuladas pela AMIB e submetidas à apreciação do Fórum Permanente Mercosul para o Trabalho em Saúde. [Internet]. São Paulo: AMIB; 2007 [citado em 2017 nov 1]. Disponível em: https://goo.gl/Cm6EM2

7. Fleck MPA. O instrumento de avaliação de qualidade de vida da Organização Mundial da Saúde (WHOQOL-100): características e perspectivas. Ciênc saúde coletiva. 2000;5(1):33-8.
8. Sintaxe SPSS. WHOQOL-bref Questionnaire. [citado em 2017 mar 12]. Disponível em: https://goo.gl/83LYp6

9. Barros DS, Tironi MOS, Nascimento CL, Neves FS, Bitencourt AGV, Almeida AM, et al. Intensive care unit physicians: socio-demographic profile, working conditions and factors associated with burnout syndrome. Rev bras ter intensiva. 2008;20(3):235-40.

10. Bezerra GKA. Unidade de terapia intensiva - perfil das admissões: Hospital Regional de Guarabira, Paraíba, Brasil. Rev Bras Ciên Saúde. 2012;16(4):491-6.

11. Barros MMS, Almeida SP, Barreto ALP, Faro SRS, Araújo MRM, Faro A. Síndrome de Burnout em médicos intensivistas: estudo em UTIs de Sergipe. Temas psicol. 2016;24(1):377-89.

12. Lourenção LG, Moscardini AC, Soler ZASG. Health and quality of life of medical residents. Rev Assoc Med Bras. 2010;56(1):81-91.

13. Lourenção LG, Moscardini AC, Soler ZASG. Qualidade de vida de residentes não médicos. Rev enferm UFPE on line. 2013;7(11):6336-45.

14. Torres AR, Ruiz T, Müller SS, Lima MCP. Quality of life, physical and mental health of physicians: a self-evaluation by graduates from the Botucatu Medical School - UNESP. Rev bras epidemiol. 2011;14(2):264-75.

15. Barbosa FT, Eloi RJ, Santos LM, Leão BA, Lima FJC, Sousa-Rodrigues CF. Correlation between weekly working time and burnout syndrome among anesthesiologists of Maceió-AL. Rev Bras Anestesiol. 2017;67(2):115-21.

16. Monteiro JK, Oliveira ALL, Ribeiro CS, Grisa GH, Agostini N. Adoecimento psíquico de trabalhadores de unidades de terapia intensiva. Psicol Ciênc Prof. 2013;33(2):366-79.

17. Fogaça MC, Carvalho WB, Nogueira-Martins LA. Preliminary study about qualify of life of physicians and nurses working in pediatric and neonatal intensive care units. Rev esc enferm USP. 2010;44(3):708-12.

18. Azevedo ALS, Silva RA, Tomasi E, Quevedo LA. Doenças crônicas e qualidade de vida na atenção primária à saúde. Cad Saúde Pública. 2013;29(9):1774-82.

19. Zortéa K. Quality of life in chronic diseases. Arq Bras Cardiol. 2010;94(5):703.

20. Freire MN, Costa ER. Qualidade de vida dos profissionais de enfermagem no ambiente de trabalho. Rev Enferm Contemp. 2016;5(1):151-8.

21. Maciel MED, Oliveira FN. Qualidade de vida do profissional técnico de enfermagem: a realidade de um hospital filantrópico em Dourados-MS. Rev Psicol Saúde. 2014;6(1):83-9.

\section{Como citar este artigo:}

Costa KJT, Silva DS, Porto AOS, Pinheiro FGMS, Souza GMR, Bonfim NSC, Nogueira EEF, Araújo DC. Qualidade de vida de médicos intensivistas: um estudo transversal. Rev. Aten. Saúde. 2017;15(54):44-48. 